\title{
MPTP-Induced Parkinsonism in the Monkey: Neurochemical Pathology, Complications of Treatment and Pathophysiological Mechanisms
}

\author{
A.R. Crossman, C.E. Clarke, S. Boyce, R.G. Robertson and M.A. Sambrook
}

\begin{abstract}
MPTP induces parkinsonism in monkeys by destruction of the substantia nigra, pars compacta. It can also damage ventral tegmental dopamine neurones and the noradrenergic locus coeruleus, both of which may be affected in Parkinson's disease. Motor symptoms in MPTP-treated monkeys respond readily to levodopa or dopamine agonist therapy. Administration of levodopa over 4-8 weeks leads to the emergence of "peak-dose" dyskinesia. Such abnormal movements are not seen following challenge doses of levodopa in animals not on long-term therapy. Radioligand studies reveal a 40-180\% increase in D2 receptor binding in the striatum of parkinsonian monkeys. 2-deoxyglucose studies of regional brain metabolism indicate that MPTP-induced parkinsonism is characterised by abnormally increased activity of medial pallidal neurones which project to the thalamus and pedunculopontine nucleus and reduced activity of subthalamic nucleus neurones.
\end{abstract}

\begin{abstract}
RÉSUMÉ: Parkinsonisme induit par le MPTP chez le singe: pathologie, neurochimie, complications du traitement et mécanismes pathophysiologiques. L'administration de MPTP provoque le parkinsonisme chez le singe, en détruisant la substance noire et en particulier la partie compacte. Il peut également nuire aux neurones dopaminérgiques du tégmentum et aux neurones noradrénérgiques du locus cinereus, lesquels sont souvent atteints dans la maladie de Parkinson. Les symptômes moteurs chez les singes traités de MPTP répondent bien au traitement aux agonistes de lévodopa ou de dopamine. L'administration de lévodopa au cours de 4 à 8 semaines entraîne l'émergence d'une dyskinésie à la «dose au sommet». Les tels mouvements anormaux ne se trouvent pas à la suite des doses provocatrices de lévodopa chez les animaux qui reçoivent la thérapie à court terme. Des études de liason au moyen de radioligands révèlent une augmentation de 40 à 80 pour 100 dans la liason aux récepteurs dopaminérgiques de type D2 dans le striatum des singes parkinsoniens. Des études du métabolisme cérébral régional, à l'aide du 2-désoxyglucose marqué, indiquent que le parkinsonisme provoqué par le MPTP est caractérisé par une activité accrue de façon anormale au niveau des neurones du globus pallidus qui projettent vers le thalamus et au noyau pédonculopotin, et aussi par une activité réduite des neurones du noyau sous-thalamique.
\end{abstract}

Can. J. Neurol. Sci. 1987; 14:428-435

\section{Clinical Features}

It is well-established that the neurotoxin N-methyl-4-phenyl1,2,3,6-tetrahydropyridine (MPTP) can induce a profound and permanent parkinsonian state when administered to man., The clinical picture is virtually indistinguishable from idiopathic Parkinson's disease and postencephalitic parkinsonism, being dominated by profound hypokinesia and bradykinesia, rigidity, and both postural and resting tremor. Other symptoms associated with the idiopathic and postencephalitic conditions are also encountered, such as micrographia, hypophonia, seborrhea, drooling and kinesia paradoxica. ${ }^{1,2,3,4}$. These patients have been found to respond well - at least at first - to conventional antiparkinson therapy with L-dihydroxyphenylalanine(L-DOPA) or bromocriptine.

Soon after its effects in man had been recognised it was shown that systemic administration of MPTP to monkeys induces a parkinsonian state similar to that observed in man. ${ }^{5.6,7}$ The animals progressively develop marked hypo- and bradykinesia, rigidity and postural tremor. True resting tremor was apparently not a prominent feature of the condition, according to early reports. It now seems generally agreed, however, that both resting and postural tremor may co-exist in affected animals. In severe cases the monkeys assume a hunched, flexed attitude, with loss of postural fixation of the head, reminiscent of the "somersault" or "pallidal" posture which has been described following bilateral lesions of the globus pallidus. ${ }^{8,9,10}$ An essentially similar condition has been reported to develop following MPTP administration in a number of species, including the rhesus monkey, ${ }^{5,7}$ squirrel monkey, ${ }^{11}$ cynomolgus monkey ${ }^{12,13}$ and marmoset. ${ }^{14}$ The condition in experimental primates responds readily, and in a dose-dependent fashion, to L-DOPA or dopamine agonists $5,7,15$ which completely reverse the motor deficit.

From the Department of Cell \& Structural Biology, School of Biological Sciences, Stopford Building, University of Manchester, Manchester

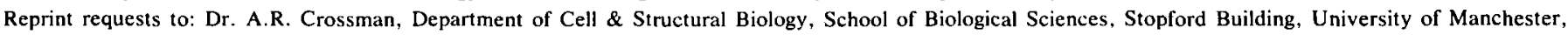
Manchester, U.K. M13 9PT 
There has been debate concerning the stability of parkinsonism induced by MPTP in the monkey, which is highly relevant to its usefulness as a model of the human condition. Dramatic recovery appears to occur following the cessation of administration in some species (for example the marmoset) and with certain administration schedules. Our own experience with a colony of cynomolgus monkeys, studied for up to 15 months after MPTP administration, is that a variable degree of gradual recovery from disability occurs within the first few weeks following exposure to the toxin. After this the residual parkinsonian features, which may be mild or severe depending upon the dose administered and individual variation between animals, remains essentially stable, with no appreciable further recovery or deterioration. This renders the model ideally suited to long-term studies of drug therapy and chronic changes in brain function.

\section{Pathological Findings}

Postmortem examination of the brain of one human subject exposed to MPTP showed destruction of the pigmented dopaminergic cells of the substantia nigra, pars compacta.' Although in life this patient had abnormally low cerebrospinal fluid (CSF) levels of homovanillic acid (HVA), 5-hydroxyindoleacetic acid (5-HIAA) and noradrenaline, which suggest abnormalities of central dopaminergic, serotonergic and noradrenergic systems, structural damage appeared to be restricted to the substantia nigra. The locus coeruleus and ventral tegmental area were reported to be intact.

Similarly, MPTP-induced parkinsonism in the monkey is associated $w$ th destruction of nigral dopaminergic neurones, together with depletion of striatal dopamine levels. ${ }^{4,5,6,11}$ The ventral tegmental area, from which arises the dopaminergic innervation of the nucleus accumbens, has generally been reported to be intact ${ }^{5,6,16}$ as has the locus coeruleus. These are important observations since they argue strongly a) that the severe parkinsonian symptoms observed in both man and monkey are due solely to destruction of the substantia nigra and b) that MPTP is a uniquely-specific nigral toxin.

In subsequent studies, in the cynomolgus monkey, a less specific action of MPTP was revealed. Mitchell et al ${ }^{13}$ reported that in monkeys with severe parkinsonism (judged to be the simian equivalent of stages IV-V on the Hoehn and Yahr ${ }^{21}$ parkinsonian rating scale for man) the neurotoxic action of MPTP was not necessarily entirely selective for substantia nigra neurones. In some monkeys it additionally caused a) destruction of cells in the ventral tegmental area, with associated depletion of dopamine from the nucleus accumbens, and b) profound degenerative changes in the locus coeruleus, with depletion of forebrain noradrenaline (Figure 1). Even in animals with no obvious structural damage in the ventral tegmental area or locus coeruleus, catecholamine levels in their terminal projection fields were severely depleted, suggesting sub-lethal toxicity. It was proposed that MPTP exhibits a spectrum of toxicity to aminergic neurones, with a rank order of substantia nigra $>$ ventral tegmental area $>$ locus coeruleus. Further, it was pointed out that these results indicated an even closer relationship than hitherto envisaged between MPTP-induced parkinsonism in the monkey and the idiopathic condition in man, since both the ventral tegmental area and the locus coeruleus have been reported to be variably affected in the human condition.
It was suggested that the variance of these neuropathological observations from those of other studies might reflect differences between species or the doses of MPTP administered. Subsequently, MPTP-induced degenerative changes in the locus coeruleus, have been confirmed by other workers. ${ }^{17}$

The conclusion from the above clinical and neuropathological observations is that MPTP-induced parkinsonism in the monkey is undoubtedly the best available model of Parkinson's disease in man. All of the major motor abnormalities of the condition are faithfully reproduced as are the pre-eminent pathological changes. The condition is essentially stable over long periods. This model therefore offers a unique opportunity for experimental studies on a) the long-term complications associated with the treatment of Parkinson's disease and b) the neural mechanisms which mediate parkinsonian symptoms.

\section{L-Dopa and Dopamine Agonist-Induced Dyskinesia}

In a significant proportion (60-80\%) of parkinsonian patients on long-term L-dopa therapy there may develop a number of complications to effective management, one of the most common being "peak-dose" dyskinesia. ${ }^{18}$ The physical manifestations of dyskinesia may vary considerably, being of a choreic, athetoid, ballistic, dystonic or myoclonic character. In some patients the dose of therapeutic agent which induces these troublesome side-effects may be below that necessary for stable management of parkinsonian symptoms, in which case they become a dose-limiting factor. The brain mechanisms which mediate dyskinesias, and the strategies by which they may be overcome, are far from understood and are, therefore, the subject of considerable interest.

It has been suggested that chronic exposure to L-dopa in itself might increase the predisposition towards the development of treatment-related complications. ${ }^{19.20}$ This possibility has prompted some authorities to advocate delaying the use of L-dopa until absolutely necessary, and then only at minimal therapeutic doses, in the belief that this approach will maximise the value to be gained from this form of treatment. Others have held that the severity of parkinsonism and the duration of disease are the main factors in determining the likelihood of treatment-related side-effects, rather than the duration of treatment per se. Despite considerable debate, however, the question remains unresolved, mainly because of the difficulty of interpreting human data. Clearly, a realistic primate model of L-dopa-induced dyskinesia would be of enormous potential value from two points of view. Firstly, it would permit experimental studies to be undertaken in order to define the abnormal neural mechanisms in operation in dyskinesias and the factors involved in their development and, secondly, it would provide an ideal testing-ground for new strategies designed to combat dyskinesia.

We have studied the effects of regular and intermittent L-dopa and dopamine agonist therapy in a group of monkeys for up to 15 months after the induction of parkinsonism with MPTP. We can now report that dyskinesia, closely resembling that seen in man, can readily be induced in these animals.

MPTP, at a dose of $0.4-0.57 \mathrm{mg} / \mathrm{kg}$, was administered intravenously to six cynomolgus monkeys (Macaca fascicularis) of mixed sex at 7-14 day intervals. The gradual progression of parkinsonian symptoms was carefully monitored until it reached approximately stage IV on the Hoehn and Yahr rating scale for man. ${ }^{21}$ This was achieved with total cumulative doses ranging 


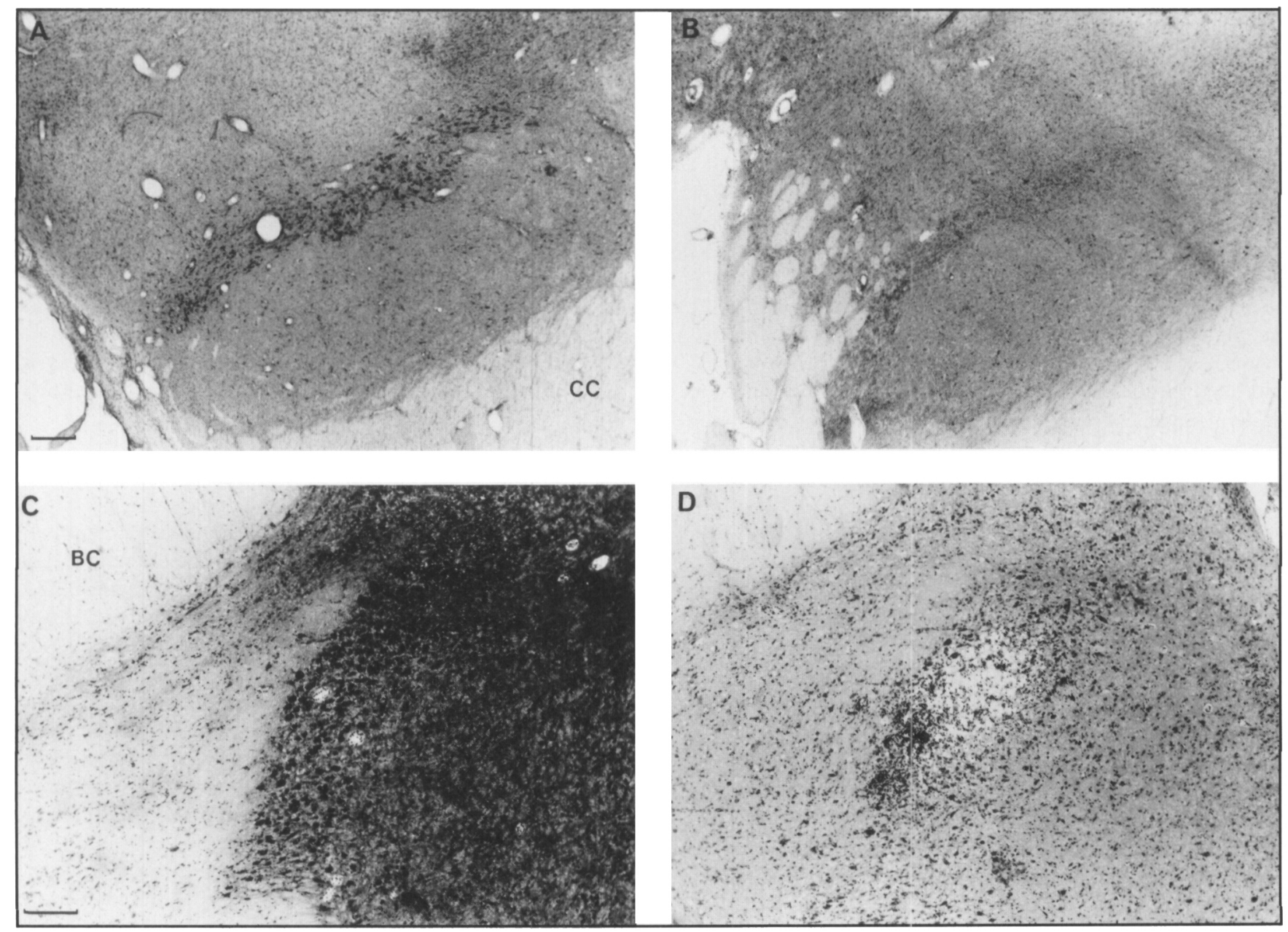

Figure I-Photomicrographs showing the substantia nigra $(A$ and $B)$ and the locus coeruleus $(C$ and $D)$ of control $(A$ and $C)$ and $M P T P$-treated $(B$ and $D)$ monkeys. Note loss of substantia nigra, pars compacta neurones in $B$ and destruction of the locus coeruleus in D. Abbreviations: CC, crus cerebri; $B C$, brachium conjunctivum. Calibration bars: in A for substantia nigra, approximately 400 um; in C for locus coeruleus, approximately 200 um.

from $2.6-27.0 \mathrm{mg} / \mathrm{kg}$. Six to twenty-eight weeks after the last dose of MPTP, when parkinsonian symptoms were demonstrably stable, regular, thrice-daily oral administration of therapeutically-active doses of L-dopa ( $100 \mathrm{mg} \mathrm{L}$-dopa plus $10 \mathrm{mg}$ peripheral decarboxylase inhibitor) were commenced in three of the monkeys. The response of all six monkeys to test doses of L-dopa or apomorphine $(0.1 \mathrm{mg} / \mathrm{kg})$ were examined at twoweek intervals.

Initially, all animals showed a good clinical response to $L$-dopa, which abolished all motor manifestations of parkinsonism. At the doses used, neither L-dopa nor apomorphine induced dyskinesia in any animal. However, within 4-8 weeks of regular treatment, all three monkeys receiving L-dopa began to develop dyskinetic movements after each dose (Figure 2). Similar abnormal movements were observed following administration of apomorphine. In humans exposed to MPTP, complications of therapy, including dyskinesias, have likewise been reported to emerge at an early stage. ${ }^{4}$ In the monkey, dyskinesia was first observed in the lower limbs, which exhibited intermittent choreoathetoid movements. With the further passage of time, during which regular L-dopa administration was maintained, the dyskinesia became more severe, more continuous, of longer duration, and spread to involve the upper limbs and the orofacial musculature. Dyskinesia was quantifiable and dose-dependent (Figures 3 and 4). Dyskinesia of the lower limbs remained the most prominent throughout. It typically took the form of complex combinations of alternating plantar- and dorsiflexion of the foot, eversion/inversion of the foot, flexion/extension of the knee, flexion/extension of the hip, and adduction/abduction of the hip.

We propose that such manifestations of abnormal involuntary movements are the simian equivalent of "peak-dose" dyskinesia in man, which emphasises still further the close relationship between the characteristics of MPTP-induced parkinsonism in the monkey and Parkinson's disease in man. When severe, the choreic limb movements induced by L-dopa or dopamine agonists in MPTP-treated monkeys were virtually indistinguishable from hemiballismus in these animals which we have shown, in previous studies, may be produced by injection of antagonists of the transmitter gamma-aminobutyric acid (GABA) into the subthalamic nucleus. ${ }^{22.23}$ In addition to chorea, however, L-dopa or dopamine agonist-induced dyskinesia often had a prominent dystonic component, which first became obvious after an initial period of choreic activity. It appeared to correspond to the period of maximal effect of drug, although this remains to be proven. Dystonia was usually mani- 


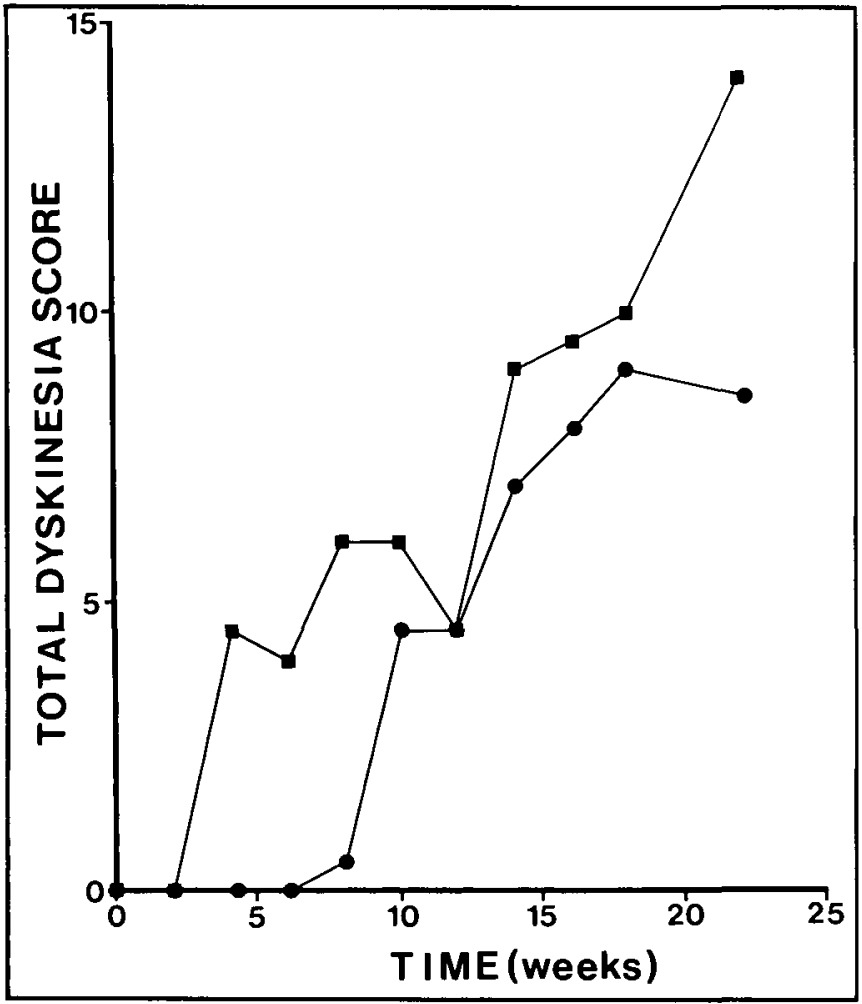

Figure $2-$ Graph showing the influence of regular $L$-dopa medication on the appearance of dyskinesia following single doses of apomorphine 10.1 $\mathrm{mg} / \mathrm{kg}$, i.m.) in two MPTP-treated monkeys, as described in the text. Dyskinesia was scored on an arbitrary scale of $0-3$ where $0=$ no dyskinesia, $l$ = intermittent, 2 = continuous mildor moderate dyskinesia, $3=$ continuous severe dyskinesia (seriously interfering with normal activities). Dyskinesia was assessed separately in the face, upper and lower limbs at 5 minute intervals and scores were totalled over 90 minutes.

fest by prolonged periods (many minutes) of tonic flexion of the hip and knee which brought the knee up to the chin, torticollis, retrocollis and truncal twisting.

Dyskinesia was not observed following administration of L-dopa or dopamine agonists to monkeys which were not receiving chronic L-dopa medication, even though the duration and severity of their parkinsonism was indistinguishable from that of animals in the treated group. These data suggest that exposure to L-dopa increased the predisposition of treated animals to develop dyskinesia.

\section{Ligand Binding Studies}

It is generally accepted that in lower animals destruction of the nigrostriatal dopaminergic pathway leads to the development of dopamine receptor supersensitivity in the denervated striatum. Until recently the data relating to the status of dopamine receptors in the human striatum in Parkinson's disease has not been so clear cut. Guttman and Seeman ${ }^{24}$ have, however, provided convincing evidence of increased $\mathrm{D} 2$ dopamine receptor density in the striata of non-treated parkinsonian patients. The few reports which deal with dopamine receptors in the MPTP-treated monkey are as confusing as those in man. Thus, Jenner et $\mathrm{al}^{14}$ were unable to detect any change is specific binding of $[3 \mathrm{H}]$-spiperone in the caudate-putamen of MPTPtreated marmosets, while Bedard et $\mathrm{al}^{25}$ apparently found a decrease in the cynomolgus monkey. In a single cynomolgus monkey, made hemiparkinsonian by unilateral internal carotid

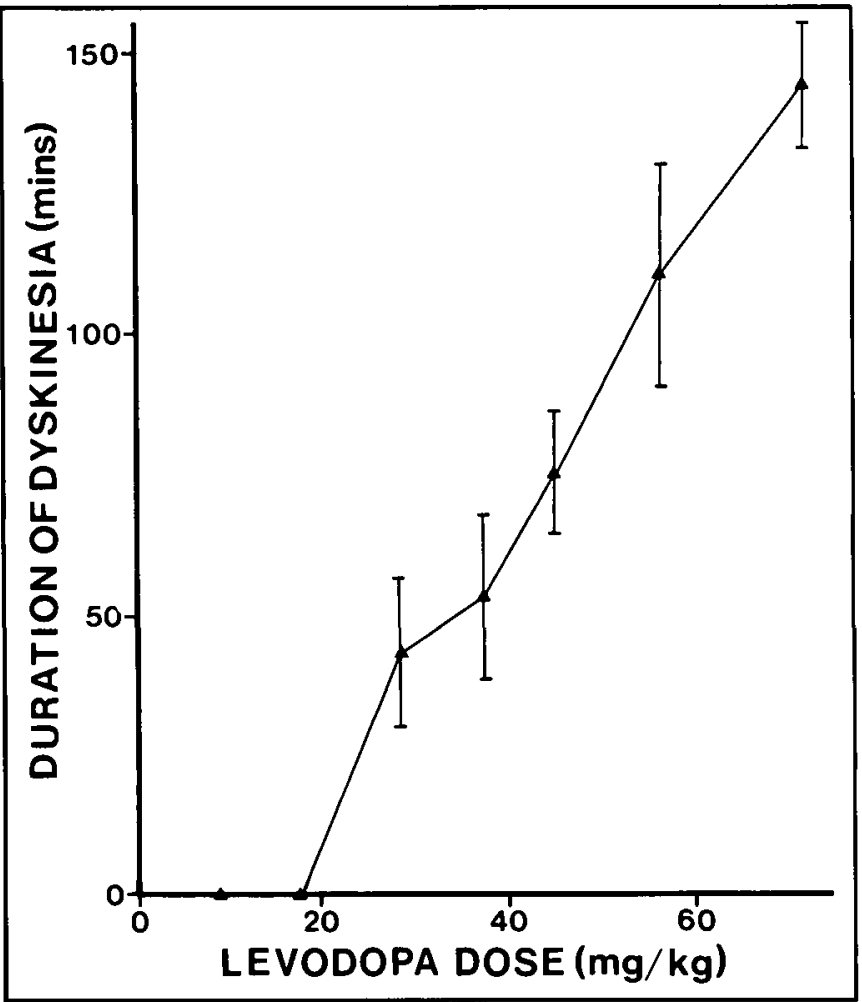

Figure 3 - Graph showing the effect of increasing doses of L-dopa on the duration of dyskinesia in an MPTP-treated monkey. Data were obtained after approximately 3 months L-dopa therapy (75 $\mathrm{mg}$ t.d.s.). Results represent mean \pm S.E.M. of three trials.

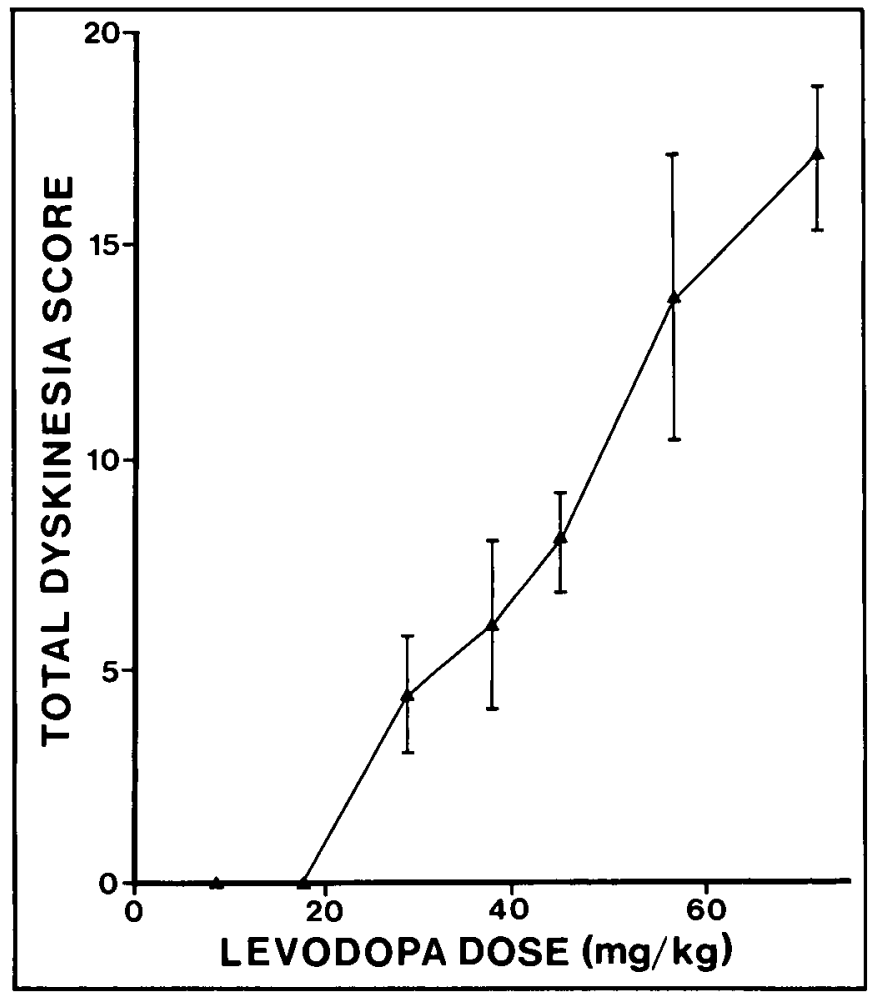

Figure 4 - Graph showing the relationship between dose of L-dopa administered and "total dyskinesia score" for an MPTP-treated monkey after 3 months of L-dopa therapy ( $75 \mathrm{mg}$ t.d.s.). Dyskinesia was scored on an arbitrary scale of $0-2$, where $0=$ no dyskinesia, $I=$ mild, $2=$ severe dyskinesia (seriously interfering with normal activities). Dyskinesia was assessed at 10 minute intervals and scores were totalled over a three hour trial period. Results represent mean \pm S.E.M. of three trials. 
artery infusion of MPTP, Joyce et al, ${ }^{26}$ using autoradiographic techniques, reported increased spiperone binding in the lateral caudate and putamen.

We have examined the effects of MPTP treatment on the regional distribution of specific [3H]-spiperone binding in brains of four cynomolgus monkeys, using in vitro semi-quantitative autoradiography. The monkeys received daily intravenous injections of MPTP over 7-8 days to a total cumulative dose of 2.79 $3.85 \mathrm{mg} / \mathrm{kg}$. Animals were killed $15-20$ days later by barbiturate overdose and the brains were immediately frozen in isopentane. Three untreated animals served as controls. Tissue was blocked stereotactically and 20um sections were thaw-mounted on gelatincoated glass slides. Histological and neurochemical evaluation revealed profound cell loss from the substantia nigra, pars compacta and ventral tegmental area, together with severe depletion of striatal and accumbens dopamine levels, as previously reported. ${ }^{13}$

Sections for binding studies were washed twice in phosphate buffer $(0.1 \mathrm{M}), \mathrm{pH} 7.4$, for $30 \mathrm{~min}$ at $20^{\circ} \mathrm{C}$. The sections were incubated at $20^{\circ} \mathrm{C}$ for $60 \mathrm{~min}$ in $50 \mathrm{mM}$ Tris- $\mathrm{HC} 1, \mathrm{pH} 7.4$, containing $120 \mathrm{mM} \mathrm{NaCl}$ to which had been added $0.3 \mathrm{nM}[3 \mathrm{H}]$-spiperone (specific activity $76 \mathrm{Ci} / \mathrm{mmol}$ ) and $0.1 \mathrm{uM}$ ketanserin (to mask binding to 5HT-2 sites). Specific binding was defined as that displaced by luM haloperidol. Following incubation sections were washed in ice-cold buffer for $2 \times 1 \mathrm{~min}$, air-dried and co-exposed to $[3 \mathrm{H}]$-sensitive film with tritium standards (Amersham) for 34 days. Autoradiographs ( 2 of total binding and 1 of non-specific binding for multiple brain levels in each animal) were analysed using a computer-based image analysis system. In order to determine the topographical distribution of $[3 \mathrm{H}]$-spiperone binding the caudate and putamen were divided into subregions for analysis.

Specific $[3 \mathrm{H}]$-spiperone binding in control monkey brains showed a heterogeneous distribution within the basal ganglia. The highest levels of binding were found in the caudate and putamen, only moderate binding being present in the nucleus accumbens, and very little in the globus pallidus. Within the putamen, there was a heterogeneous distribution of binding which showed a marked medial to lateral increase. The highest levels of binding were found in the dorsolateral part of putamen at rostral levels and the ventrolateral part of the putamen at caudal levels. The caudate displayed a more even distribution.

Autoradiographs from MPTP-treated monkeys demonstrated a marked enhancement $(40-180 \%)$ in specific $[3 \mathrm{H}]$-spiperone binding in the caudate and putamen compared to control animals (Figure 5, Table 1). In some, but not all, autoradiographs the greatest increase appeared to be associated with the lateral portion of the striatum, as described by Joyce et $\mathrm{al}^{26}$ in a unilaterally lesioned animal. No change in specific binding was found in the nucleus accumbens, despite the equally dramatic depletion of dopamine from this area.

\section{Regional 2-Deoxyglucose (2-DG) Uptake}

The MPTP-treated monkey provides a unique opportunity for studying the abnormal brain mechanisms which are responsible for the appearance of parkinsonian symptoms. One approach by which this may be achieved is by application of the 2-DG uptake technique described by Sokoloff et al. ${ }^{27}$ Radioactively labelled 2-DG is administered systemically and its regional uptake by the brain is measured autoradiographically. Differential labelling between regions indicates differences in metabolic

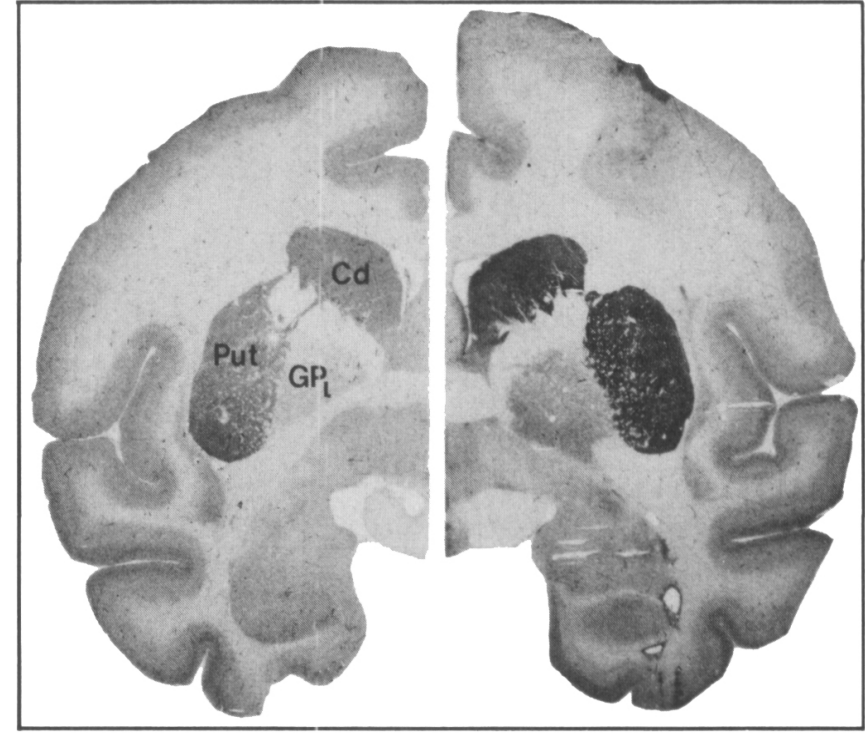

Figure 5 - Autoradiographs showing specific binding of [3H]-spiperone to the brain of a normal monkey (left) and an MPTP-treated monkey. Binding study was performed as described in the text. Note the increased binding in the caudate nucleus, putamen and lateral segment of the globus pallidus. Abbreviations: $C d$, caudate nucleus; GPl, lateral segment of the globus pallidus; Put, putamen.

Table 1: Specific Binding of [3H]-Spiperone to Basal Ganglia in Control and MPTP-Treated Monkey Brains

\begin{tabular}{|c|c|c|}
\hline \multirow[t]{2}{*}{ Brain region } & \multicolumn{2}{|c|}{ [3H]-spiperone binding (fmol/mg tissue) } \\
\hline & Control & MPTP \\
\hline Nucleus Accumbens & $22.8(n=2)$ & $25.9 \pm 7.1$ \\
\hline Caudate Nucleus & $26.3 \pm 3.5$ & $67.4 \pm 11.8^{*}$ \\
\hline Putamen & $27.1 \pm 1.2$ & $73.6 \pm 6.5^{*}$ \\
\hline
\end{tabular}

Values are expressed as the mean specific binding $( \pm 1$ SEM) for 3 monkeys in each group.

${ }^{*} \mathrm{p}<0.05$ compared to control values (Student's t-test).

activity and, thus, neuronal electrical activity. 2-DG is accumulated by the same membrane transport system as glucose, and is phosphorylated by hexokinase, the first enzyme in oxidative metabolism. ${ }^{27}$ Following phosphorylation, 2-DG is essentially trapped in the cell and does not undergo significant further metabolism. It is argued that since in activated neurones, the majority of the increase in energy requirement is associated with the ouabain-sensitive sodium pump, ${ }^{28}$ and that since this energy requirement is greater for structures with a large surface:volume ratio (such as axon terminals) than for those with a small surface:volume ratio (such as neuronal perikarya), ${ }^{28,29}$ then regional changes in 2-DG uptake, which occur as a result of experimental manipulation, are probably largely due to changes in the activity of nerve terminals within the affected structure, rather than primarily reflecting the activity of intrinsic neuronal cell bodies.

We have applied the 2-DG uptake technique to monkeys made parkinsonian either by generalised intravenous administration of MPTP or following unilateral intracarotid infusion. The 2-DG procedure was performed up to 4 weeks after administration of MPTP in the bilaterally-affected animals and 3-11 weeks post-MPTP in the unilaterally-lesioned animals. 
Marked changes were found in the regional uptake of 2-DG in the brains of parkinsonian animals. ${ }^{12,30,31}$ In animals with generalised parkinsonism there was a profound increase in 2-DG uptake by the globus pallidus, which was most marked in the lateral segment. There were also marked increases in uptake by the VA/VL thalamic nuclei and by the pedunculopontine nucleus. The subthalamic nucleus alone displayed a prominent and consistent decrease in 2-DG uptake. The pattern of 2-DG uptake in hemiparkinsonism was similar, but not identical, to that observed in bilaterally affected animals (Figures 6,7 and 8). The main differences were that the increased uptake in the globus pallidus was virtually entirely restricted to the lateral segment and, in addition, the increased uptake in the thalamus was less obvious.

The increased uptake of 2-DG by the VA/VL thalamus and pedunculopontine nucleus are interpreted as indicating abnormal overactivity of cells in the medial pallidal segment, which project to these structures. The 2-DG changes in these nuclei were, in fact, the reverse of those seen in experimental models of hyperkinetic disorders, such as hemiballismus and chorea, which have been the subject of previous studies in this laboratory, ${ }^{31,32,33}$ and which, it is believed, are characterised by abnormal underactivity of medial pallidal neurones. ${ }^{31}$ The increased uptake of 2-DG found in the globus pallidus itself was probably due to increased activity in its afferent pathways, the most likely candidates being projections from the subthalamic nucleus and the neostriatum. The change in 2-DG uptake observed in the subthalamic nucleus is entirely consistent with this view. Thus, decreased uptake in the subthalamic nucleus most likely indicates decreased activity of its own afferent pathways, the most massive of which is from the lateral segment of the globus pallidus. Since this pathway is thought to be GABAergic and inhibitory upon subthalamic cells a decrease in its level of activity would lead to disinhibition of subthalamic cells, which in turn project heavily upon both segments of the globus pallidus.

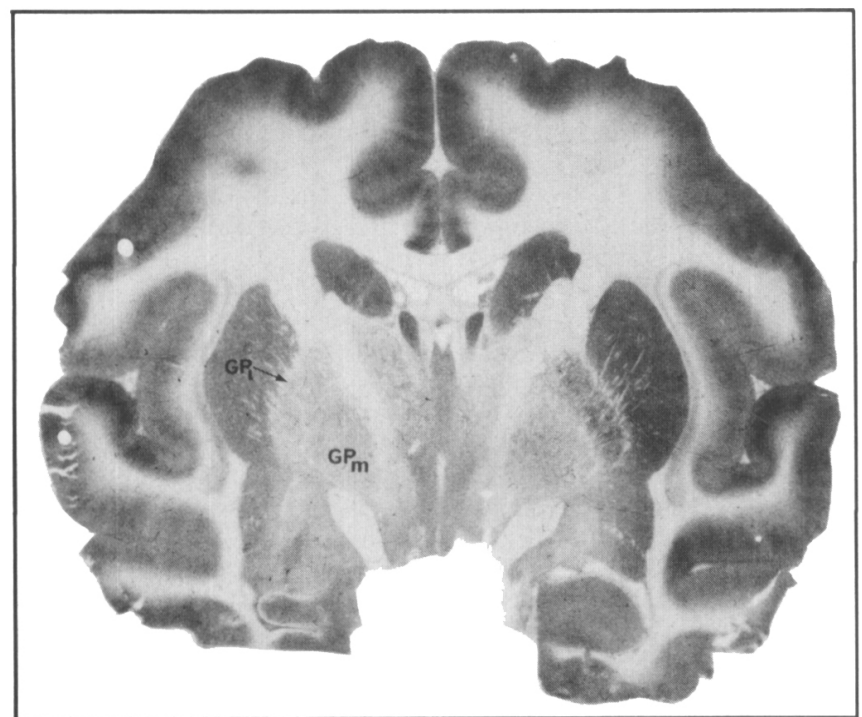

Figure 6 - Autoradiograph showing regional uptake of 2-deoxyglucose in the basal ganglia of a monkey 3 months after induction of unilateral parkinsonism by infusion of MPTP into the right internal carotid artery. Note the increased uptake of 2-DG in the globus pallidus of the lesioned side. Abbreviations: GPl, lateral segment of the globus pallidus; GPm, medial segment of the globus pallidus.
This might, therefore, in part account for the increased uptake of 2-DG by the globus pallidus.

Equally as important as the detailed neural mechanisms, however, is the principle clearly emerging from 2-DG studies, that the subthalamic nucleus is implicated in the pathophysiology of parkinsonism. This is an entirely new concept which, if correct, puts this nucleus at the centre of the neural mechanisms of a spectrum of movement disorders, ranging from hemiballismus and chorea to parkinsonism.

\section{SUMMARY}

1. MPTP-induced parkinsonism in the monkey is the best available model of idiopathic Parkinson's disease in man. It

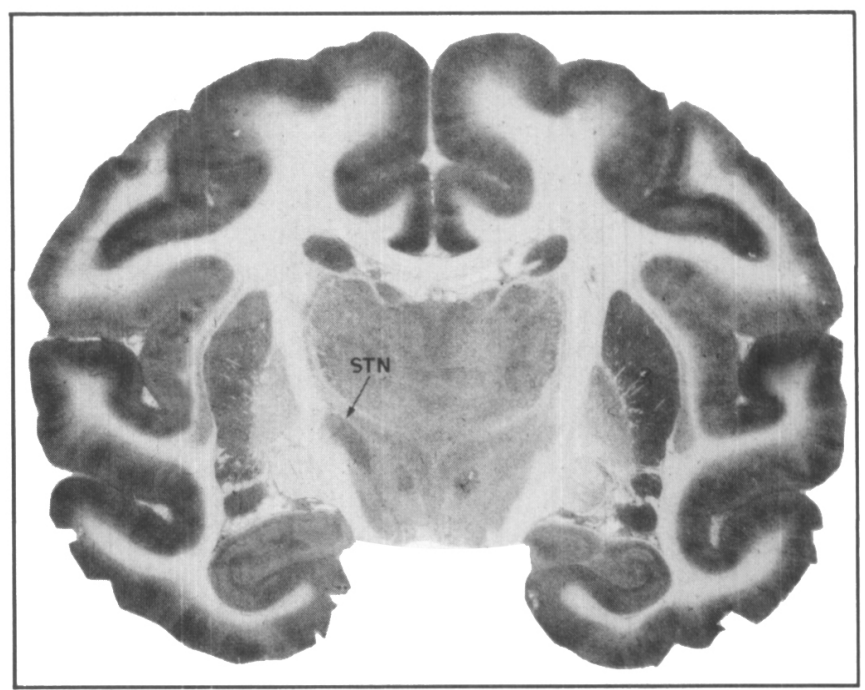

Figure 7 - Autoradiograph showing regional uptake of 2-deoxyglucose in the diencephalon of a monkey 3 months after induction of unilateral parkinsonism by infusion of MPTP into the right internal carotid artery. Note the decreased uptake of 2-DG by the subthalamic nucleus (STN) on the lesioned side.

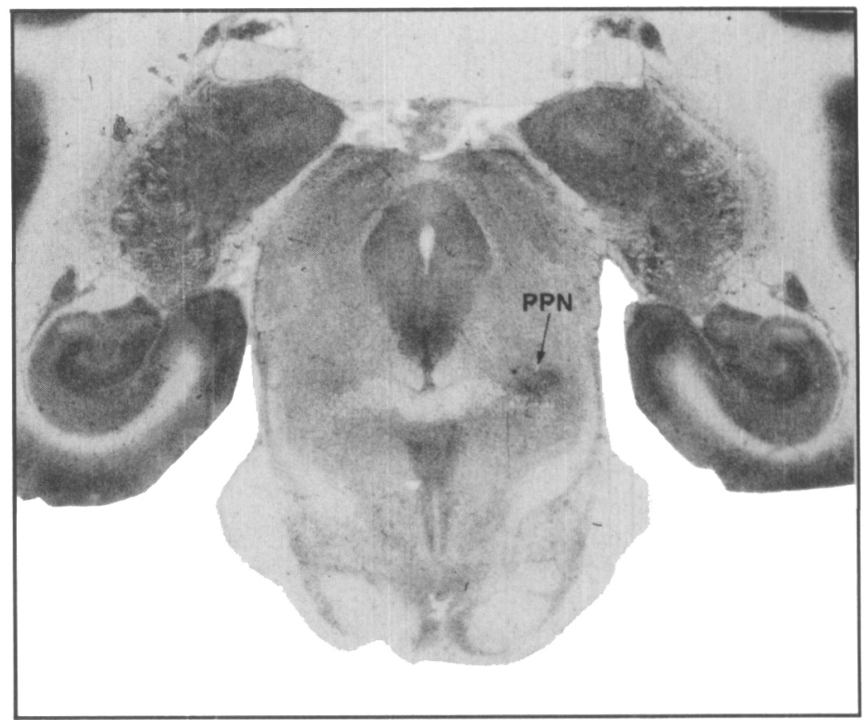

Figure 8-Autoradiograph showing regional uptake of 2-deoxyglucose in the brainstem of a monkey 3 months after induction of unilateral parkinsonism by infusion of MPTP into the right internal carotid artery. Note the increased uptake by the pedunculopontine nucleus (PPN) on the lesioned side. 
reproduces all of the major motor abnormalities (hypokinesia/ bradykinesia, rigidity and tremor) and the pre-eminent neuropathology (degeneration of the substantia nigra, pars compacta) of the human condition.

2. MPTP administration to the monkey may also induce degeneration of the dopaminergic cells of the ventral tegmental area and the noradrenergic cells of the locus coeruleus. Since these cell groups are often involved in the pathology of human Parkinson's disease, this suggests that the correlation between MPTP-induced parkinsonism in the monkey and the human condition may be closer than previously envisaged.

3. MPTP-induced parkinsonism in the monkey is stable over long periods (at least 15 months), making it ideally suited to long-term experimental studies.

4. Regular administration of L-dopa to parkinsonian monkeys leads to the rapid development of what appears to be the simian equivalent of "peak-dose" dyskinesia. Untreated monkeys do not appear to develop this complication, suggesting that L-dopa itself may increase the predisposition towards dyskinesia.

5. There is a substantial (40-180\%) increase in D2 dopamine receptor binding in the striatum of MPTP-treated monkeys.

6. 2-deoxyglucose uptake studies suggest that in MPTP-induced parkinsonism there is abnormally increased activity in the cells of the medial segment of the globus pallidus, and abnormally decreased activity in the cells of the subthalamic nucleus. The subthalamic nucleus is probably involved in the pathophysiological mechanisms which mediate parkinsonian symptoms.

\section{ACKNOWLEDGEMENTS}

Supported by grants from The Medical Research Council and Action Research for the Crippled Child.

\section{REFERENCES}

1. Davis GC, Williams AC, Markey SP, et al. Chronic parkinsonism secondary to intravenous injection of meperidine analogues. Psychiat Res 1979; 1: 249-254.

2. Langston JW, Ballard P, Tetrud JW, et al. Chronic parkinsonism in humans due to a product of meperidine-analog synthesis. Science 1983; 219: 979-980.

3. Langston JW. MPTP and Parkinson's disease. Trends in Neurosci 1985; 8: 79-83.

4. Langston JW, Ballard P. Parkinsonism induced by 1-methyl-4phenyl-1,2,3,6-tetrahydropyridine (MPTP): Implications for treatment and the pathogenesis of Parkinson's disease. Can J Neurol Sci 1984; 11: 160-165.

5. Burns RS, Chiueh CC, Markey SP, et al. A primate model of parkinsonism: selective destruction of dopaminergic neurons in the pars compacta of the substantia nigra by $\mathrm{N}$-methyl-4-phenyl1,2,3,6-tetrahydropyridine. Proc Natl Acad Sci U.S.A. 1983; 80: 4546-4550.

6. Burns RS, Markey SP, Phillips JM, et al. The neurotoxicity of 1-methyl-4-phenyl-1,2,3,6-tetrahydropyridine in the monkey and man. Can J Neurol Sci 1984; 11: 166-168.

7. Chiueh CC, Markey SP, Burns RS, et al. Selective neurotoxic effects of N-methyl-4-phenyl-1,2,3,6-tetrahydropyridine (MPTP) in subhuman primates and man: a new animal model of Parkinson's disease. Psychopharmacol Bull 1984; 20: 548-553.

8. Mettler FA. Effects of bilateral simultaneous subcortical lesions in the primate. J Neuropath Exp Neurol 1945; 4: 99-122.

9. Denny-Brown D. The Basal Ganglia and Their Relation to Disorders of Movement, Oxford Neurological Monographs (Russell WR, ed.), Oxford University Press, London, 1962.
10. Richter R. Degeneration of the basal ganglia in monkeys from chronic carbon disulphide poisoning. J Neuropath Exp Neurol $1945 ; 4: 324-353$.

11. Langston JW, Forno LS, Rebert CS, et al. Selective nigral toxicity after systemic administration of 1-methyl-4-phenyl-1,2,5,6-tetrahydropyridine (MPTP) in the squirrel monkey. Brain Res 1984; 292: 390-394.

12. Crossman AR, Mitchell IJ, Sambrook MA. Regional brain uptake of 2-deoxyglucose in N-methyl-4-phenyl-1,2,3,6-tetrahydropyridine (MPTP)-induced parkinsonism in the macaque monkey. Neuropharmacology 1985; 24: 587-591.

13. Mitchell IJ, Cross AJ, Sambrook MA, Crossman AR. Sites of the neurotoxic action of 1-methyl-4-phenyl-1,2,3,6-tetrahydropyridine in the monkey include the ventral tegmental area and the locus coeruleus. Neurosci Letts 1985; 61: 195-200.

14. Jenner P, Rupniak NMJ, Rose S, et al. 1-methyl-4-phenyl-1,2,3,6tetrahydropyridine-induced parkinsonism in the common marmoset. Neurosci Letts 1984; 50: 85-90.

15. Clarke CE, Sambrook MA, Mitchell IJ, Crossman AR. Levodopainduced dyskinesia and response fluctuations in primates rendered parkinsonian with I-methyl-4-phenyl-1,2,3,6-tetrahydropyridine (MPTP). J Neurol Sci 1987; 78: 273-280.

16. Chiueh CC, Burns RS, Markey SP, et al. Primate model of parkinsonism: selective lesion of nigrostriatal neurons by 1-methyl-4-phenyl-1,2,3,6-tetrahydropyridine produces an extrapyramidal syndrome in rhesus monkeys. Life Sci 1985; 36: 213-218.

17. Forno LS, Langston JW, Delanney LE, et al. Locus ceruleus lesions and eosinophilic inclusions in MPTP-treated monkeys. Ann Neurol 1986; 20: 449-455.

18. Marsden CD, Parkes JD, Quinn N. Fluctuations of disability in Parkinson's disease - clinical aspects. In: Movement Disorders (Marsden CD \& Fahn S, eds.), Butterworths, London, 1982: 96-122.

19. Lesser RP, Fahn S, Snider S, et al. Analysis of the clinical problems in parkinsonism and the complications of long-term levodopa therapy. Neurology 1979; 29: 1253-1260.

20. Rajput AH, Stern W, Laverty WH. Chronic low-dose levodopa therapy in Parkinson's disease: an argument for delaying levodopa therapy. Neurology 1984; 34: 991-996.

21. Hoehn MM, Yahr MD. Parkinsonism: onset, progression and mortality. Neurology 1967; 17: 427-442.

22. Crossman AR, Sambrook MA, Jackson A. Experimental hemiballismus in the baboon produced by injection of a gammaaminobutyric acid antagonist into the basal ganglia. Neurosci Letts 1980; 20: 369-372.

23. Crossman AR, Sambrook MA, Jackson A. Experimental hemichorea/hemiballismus in the monkey. Studies on the intracerebral site of action in a drug-induced dyskinesia. Brain 1984; 107: 579-596.

24. Guttman M, Seeman P. L-dopa reverses the elevated density of dopamine D2 receptors in Parkinson's diseased striatum. J Neural Transm 1985; 64: 93-103.

25. Bedard P, Di Paolo T, Falardeau P, et al. Chronic treatment with L-dopa, but not bromocriptine induces dyskinesia in MPTPtreated parkinsonian monkeys. Correlation with $[3 \mathrm{H}]$ spiperone binding. Brain Res 1986; 379: 294-299.

26. Joyce JN, Marshall JF, Bankiewicz KS, et al. Hemiparkinsonism in a monkey after unilateral internal carotid artery infusion of 1-methyl-4-phenyl-1,2,3,6-tetrahydropyridine (MPTP) is associated with regional ipsilateral changes in striatal dopamine D-2 receptor density. Brain Res 1986; 382: 360-364.

27. Sokoloff L, Reivich M, Kennedy C, et al. M. The [14C] deoxyglucose method for the measurement of local cerebral glucose utilization: theory, procedure, and normal values in the conscious and anesthetized albino rat. J Neurochem 1977; 28: 897-916.

28. Mata M, Fink DJ, Gainer H, et al. Activity-dependent energy metabolism in rat posterior pituitary primarily reflects sodium pump activity. J Neurochem 1980; 34: 213-215.

29. Schwartz WJ, Smith CB, Davidsen L, et al. Metabolic mapping of functional activity in the hypothalamo-neurohypophysial system of the rat. Science $1979 ; 205: 723-725$.

30. Mitchell IJ, Cross AJ, Sambrook MA, Crossman AR. Neural mechanisms mediating 1-methyl-4-phenyl-1,2,3,6-tetrahydropyridine 
-induced parkinsonism in the monkey: relative contributions of the striatopallidal and striatonigral pathways as suggested by 2-deoxyglucose uptake. Neurosci Letts 1986; 63: 61-65.

31. Crossman AR. Primate models of dyskinesia: The experimental approach to the study of basal ganglia-related involuntary movement disorders. Neuroscience 1987; 21: 1-40.

32. Mitchell IJ, Jackson A, Sambrook MA, Crossman AR. Common neural mechanisms in experimental chorea and hemiballismus in the monkey. Evidence from 2-deoxyglucose autoradiography. Brain Res 1985; 339: 346-350.

33. Mitchell IJ, Sambrook MA, Crossman AR. Subcortical changes in the regional uptake of [3H]-2-deoxyglucose in the brain of the monkey during experimental choreiform dyskinesia elicited by injection of a gamma-aminobutyric acid antagonist into the subthalamic nucleus. Brain 1985; 108: 421-438. 